\title{
SPEubsaúde
}

RELATO DE CASO

\section{HPV 16 em câncer de colo de útero durante a gravidez: relato de caso}

\section{HPV 16 in cervical cancer during pregnancy: case report}

José Rodolfo Teixeira da Cunha ${ }^{16}$, Lailson Oliveira de Castro ${ }^{26}$, Maria do Desterro Soares Brandão Nascimento ${ }^{3}$, Rodrigo Lopes da Silva4 ${ }^{4}$, Ana Beatriz Melo Guimarães 5

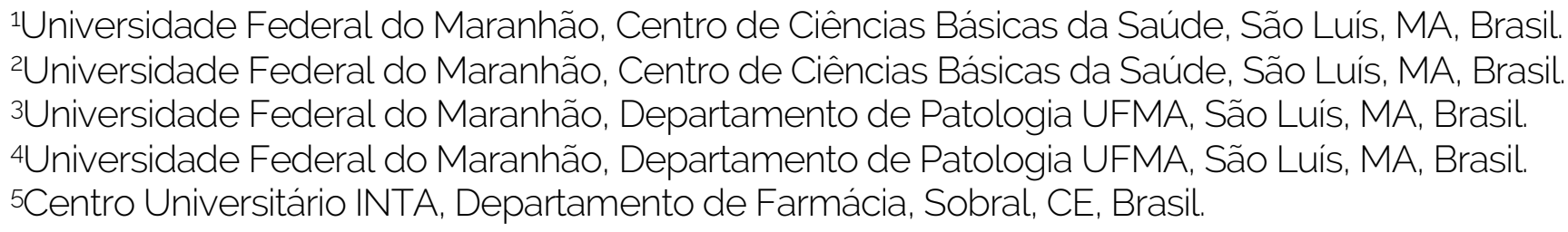

'Universidade Federal do Maranhão, Centro de Ciências Básicas da Saúde, São Luís, MA, Brasil. ¿Universidade Federal do Maranhão, Centro de Ciências Básicas da Saúde, São Luís, MA, Brasil. 3Universidade Federal do Maranhão, Departamento de Patologia UFMA, São Luís, MA, Brasil. 4Universidade Federal do Maranhão, Departamento de Patologia UFMA, São Luís, MA, Brasil. 5Centro Universitário INTA, Departamento de Farmácia, Sobral, CE, Brasil.

E-mail: joserodolfotcunha@hotmail.com

Como citar: Cunha, J.R.T., Castro, L.O., Popolim, R.S., Nascimento, M.D.S.B., Silva, R.L., Guimarães, A.B.M. 2019. HPV 16 em câncer de colo de útero durante a gravidez: relato de caso, 2, a013. DOl: https://dx.doi.org/10.31533/pubsaude2.a 013

Recebido: 03 out. 2019.

Revisado e aceito: 28 out. 2019

Conflito de interesse: os autores declaram, em relação aos produtos e companhias descritos nesse artigo, não ter interesses associativos, comerciais, de propriedade ou financeiros que representem conflito de interesse.

Licenciamento: Este artigo é publicado na modalidade Acesso Aberto sob a licença Creative Commons Atribuição 4.0 (CC-BY 4.0)
R e s u m o . 1. Introdução: o câncer de colo de útero é o principal câncer diagnosticado durante a gravidez, corresponde a 3\% dos cânceres cervicais. Devido à sua raridade e à complexidade, a padronização terapêutica é difícil e o tratamento durante a gravidez é mais desafiador ainda, pois o útero gravido é diretamente afetado durante o tratamento. 2. Relato de caso: paciente com adenocarcinoma bem diferenciado, com queixa de sangramento pós-coito há 3 anos. Durante a investigação para realização de estadiamento (ia1) em abril de 2016 no serviço de cirurgia oncológica, identificou-se gravidez de 14 semanas. 3. Discussão: diversas questões e controvérsias ainda permanecem sobre este assunto. Os fatos e as incertezas devem ser amplamente discutidos com a paciente. $\mathrm{O}$ efeito de atrasar a cirurgia, radioterapia e quimioterapia com o intuito de permitir a gravidez em tumores de colo de útero ainda não é bem esclarecido.

P a l a vr a s - c h a ve : Câncer, Colo de útero, Gravidez.

A b s t r a c t . 1. Introduction: cervical cancer is the main cancer diagnosed during pregnancy, it corresponds to $3 \%$ of cervical cancers. Considering its rarity and complexity, a difficult therapeutic standardization and treatment during pregnancy is even more challenging as the recorded uterus is directly affected during treatment. 2. Case report: patient with well-differentiated adenocarcinoma, complaining of postcoital bleeding for 3 years. During the investigation to perform staging (ia1) in april 2016 at the oncology surgery service, a 14-week pregnancy was identified. 3. Discussion: several issues and controversies still remain on this 
subject. The facts and uncertainties should be widely discussed with the patient. The effect of delaying surgery, radiotherapy and chemotherapy in order to allow pregnancy in cervical tumors is not yet well understood.

K e y w o r d s : Cancer, Cervix, Pregnancy.

\section{INTRODUÇÃO}

O câncer do colo do útero é uma das principais causas de morte por câncer entre as mulheres. No mundo, o câncer de colo do útero é o quarto mais frequente no público feminino, resulta em cerca de 530 mil novos casos por ano com 270 mil mortes. Aproximadamente $85 \%$ das mortes por câncer de colo de útero ocorrem em países subdesenvolvidos ou em desenvolvimento (Small et al., 2017). A maior parte dos casos de câncer de colo de útero resultam de infecção pelo papilomavírus humano (HPV) e cerca de $95 \%$ das lesões cervicais apresentam esse vírus. A maioria das infecções por HPV são transitórias e serão combatidas espontaneamente. Em alguns casos, a infecção resultará em condições pré-malignas no tecido intra-epitelial cervical. Sem tratamento, a transição da displasia para o carcinoma invasivo ou adenocarcinoma pode demorar anos ou décadas para se desenvolver, porém, em aproximadamente $10 \%$ das pacientes essa transição pode ocorrer em menos de 1 ano (Small et al., 2017).

O câncer de colo de útero é o principal câncer diagnosticado durante a gravidez, com uma incidência variando de 1 a 10 por 10000 gestações, correspondendo a $3 \%$ de todos os cânceres de colo de útero diagnosticados (Dawood et al., 2013). Pacientes gestantes em estágio inicial que optarem pela gestação expectante (FIGO estádio IB1) com diagnóstico em qualquer fase da gravidez não tem piora do quadro à longo prazo, enquanto que o manejo de pacientes grávidas com massas tumorais maiores (FIGO estágio $\geqslant$ IB1) é desafiador, o atraso no tratamento suscita preocupações com a morbidade materna, especialmente quando o diagnóstico ocorre no segundo trimestre (Takushi et al., 2002; Vliet et al., 1998). Os sinais clínicos da doença maligna podem ser mascarados pelas alterações fisiológicas da gravidez e as informações sobre a segurança do tratamento quimioterápico provém de estudos retrospectivos, com amostragem limitada. Além destas dificuldades, deve-se considerar que o diagnóstico e o tratamento cirúrgico, quimioterápico e radioterápico devem ponderar os riscos e os benefícios tanto para o bem-estar da mãe, quanto do feto (Salani et al., 2014). As pacientes precisam estar conscientes de que qualquer tipo de tratamento durante a gravidez é experimental. Quando é escolhida a interrupção da gravidez, o tratamento padrão com histerectomia radical 
(com feto no útero) e quimiorradiação são duas opções viáveis (Han et al., 2013).

Devido à raridade da doença e à complexidade de todos os fatores que devem ser levados em consideração, a padronização do tratamento é muito difícil. O tratamento do câncer durante a gravidez é mais desafiador ainda, principalmente no caso do câncer de colo de útero, uma vez que o útero grávido é afetado durante o tratamento. Ao tratar uma paciente grávida com câncer de colo do útero, várias questões são importantes: subtipo histológico, estágio da doença, idade gestacional, complicações obstétricas, assim como os desejos das pacientes em relação à continuação versus término da gravidez. Esses fatores devem ser levados em consideração e discutidos em uma equipe multidisciplinar, bem como com a paciente e seu parceiro, antes de decidir o plano de tratamento (Han et al., 2013). Em nosso relato de caso a paciente decidiu pela continuação da gravidez. O objetivo desse estudo foi relatar o caso de uma paciente com câncer de colo de útero durante a gravidez.

\section{RELATO DE CASO}

Mulher de 45 anos, casada, nulípara, cursou ensino médio completo, do lar, procedente e residente em São Luis-Ma. Relata que realizava preventivo anualmente desde os 15 anos, com menarca aos 9 anos, coitarca aos 16 anos, com história de 6 parceiros sexuais durante a vida, fez uso de pílulas anticoncepcionais e camisinha, ex-tabagista. Apresenta antecedentes familiares de câncer de próstata, Hipertensão arterial sistêmica, Diabetes Mellitus tipo II e cardiopatia.

Paciente com adenocarcinoma bem diferenciado com queixa de sangramento pós-coito há 3 anos, durante a investigação para realização de estadiamento (IA1) em abril de 2016 no serviço de cirurgia oncológica descobriu gravidez de 14 semanas. Decidiu pelo prosseguimento da gravidez e retardo do tratamento. Após o parto, foi iniciado tratamento cirúrgico em fevereiro de 2017 com histerectomia e ooforectomia, linfadenectomia pélvica, retroperitoneal e inguino-ilíaca. O laudo histopatológico das amostras cirúrgicas indicou adenocarcinoma moderadamente diferenciado primário do colo do útero, com metástase para ovário direito e o teste de PCR Nested deu positivo para HPV 16.

Tabela 1. Exame pré-operatório.

\begin{tabular}{lc}
\hline \multicolumn{2}{c}{ Hemograma completo } \\
\hline Eritrócitos & $4,28 \mathrm{milhões} / \mathrm{mm} 3$ \\
Hemoglobina & $12,60 \mathrm{~g} / \mathrm{dl}$ \\
Hematócrito & $39 \%$ \\
Leucócitos & $5270 / \mathrm{mm} 3$ \\
Plaquetas & 324000 \\
\hline
\end{tabular}




\section{DISCUSSÃO}

Em decorrência da tendência atual em adiar a gravidez para os últimos anos reprodutivos e o aumento dos programas de rastreio de câncer de colo de útero, o diagnóstico de câncer de colo de útero em estágios iniciais tem sido cada vez mais frequente em mulheres grávidas. A avaliação inicial da gestante com câncer de colo de útero deve incluir uma avaliação precisa da idade gestacional e exame ultrassonográfico completo do feto. Uma reunião multidisciplinar deve ser organizada com especialistas em ginecologia oncológica, medicina materno-fetal, neonatologistas e oncologistas com o objetivo de indicar o melhor tratamento (Ribeiro et al., 2013).

Em geral, o tratamento de mulheres grávidas com câncer de colo de útero invasivo é semelhante às não grávidas. Devido à ausência de ensaios clínicos randomizados, o manejo do câncer de colo de útero durante a gravidez permanece incerto e varia de acordo com o estágio do tumor e idade gestacional em que foi feito o diagnóstico. Alguns autores propõem que durante a segunda metade da gravidez, um atraso de 6 a 8 semanas até atingir a maturidade pulmonar fetal é aceitável para o início do tratamento. No entanto, quando o diagnóstico é feito no início da gravidez, as pacientes são aconselhadas a abortar, com o intuito de iniciar o tratamento o mais rápido possível (Monk \& Montz, 1992).

O prognóstico da gravidez é frequentemente afetado pelo diagnóstico do câncer. Um grande estudo mostrou que mulheres diagnosticadas com câncer de colo de útero durante a gravidez apresentam taxas mais elevadas de prematuridade iatrogênica, resultando em taxas aumentadas de baixo peso ao nascer (Dalrymple et al., 2005). Nesse relato de caso, o filho da paciente nasceu com Síndrome de Down.

O regime de tratamento neoadjuvante mais eficaz no câncer de colo de útero (Paclitaxel, ifosfamida, cisplatina) não é recomendado durante a gravidez, por conta do efeito deletério da ifosfamida no desenvolvimento dos rins do feto. Por outro lado, a cisplatina tem sido mais investigada em mulheres grávidas e relatórios indicam que a terapia com cisplatina pode ser administrada de forma segura após o primeiro trimestre (Fruscio et al., 2012).

O câncer colo de útero é o tipo de câncer mais desafiador durante a gravidez. Muitas questões e controvérsias ainda permanecem sobre este assunto. Os fatos e também as incertezas devem ser bem abordados com a paciente, buscando-se a decisão mais informada possível. O efeito de atrasar a cirurgia, radioterapia e quimioterapia com o intuito de permitir a gravidez em tumores de colo de útero ainda não é esclarecido. 


\section{REFERÊNCIAS}

Dalrymple, J. L., Gilbert, W. M., Leiserowitz, G. S., Cress, R., Xing, G., Danielsen, B. \& Lloyd, H. S. 2005. Pregnancy-associated cervical cancer: Obstetric outcomes. The Journal of Maternal-Fetal \& Neonatal Medicine, 17 (4), 269-276.

Dawood, R., Instone, M. \& Kehoe, S. 2013. Neo-adjuvant chemotherapy for cervical cancer in pregnancy: A case report and literature review. European Journal of Obstetrics \& Gynecology and Reproductive Biology, 172 (2), 205-208.

Fruscio, R., Villa, A., Chiari, S., Vergani, P., Ceppi, L., Dell’Orto, F., Dell'Anna, T., Chiappa, V., Bonazzi, C. M., Milani, R., Mangioni, C. \& Locatelli, A. 2012. Delivery delay with neoadjuvant chemotherapy for cervical cancer patients during pregnancy: A series of nine cases and literature review. Gynecologic Oncology, 126 (2), 192-197.

Han, S. N., Gziri, M. M. Calsterem, K. V. \& Amant, F. 2013. Cervical cancer in pregnant women: Treat, wait or interrupt? Assessment of current clinical guidelines, innovations and controversies. Therapeutic Advances in Medical Oncology, 5 (4), 211-219.

Monk, B. J. \& Montz, F. J. 1992. Invasive cervical cancer complicating intrauterine pregnancy: Treatment with radical hysterectomy. Obstetrics \& Gynecology, 80 (2), 199-203.

Ribeiro, F., Correia, L., Paula, T., Santana, I., Pinto, L. V., Borrego, J. \& Jorge, A. F. 2013. Cervical cancer in pregnancy: 3 cases, 3 different approaches. Journal of Lower Genital Tract Disease, 17 (1), 66-70.

Salani, R., Billingsley, C. C. \& Crafton, S. M. 2014. Cancer and pregnancy: An overview for obstetricians and gynecologists. American Journal of Obstetrics \& Gynecology, 211 (1), 7-14.

Small, J. W., Bacon, M. A., Bajaj, A., Chuang, L. T., Fisher, B. J., Harkenrider, M. M., Jhingran, A., Kitchener, H. C., Mileshkin, L. R., Viswanathan, A. N. \& Gaffney, D. K. 2017. Cervical cancer: A global health crisis. Cancer, 123 (13), 2404-2412.

Takushi, M., Moromizato, H., Sakumoto, K. \& Kanazawa, K. 2002. Management of invasive carcinoma of the uterine cervix associated with pregnancy: Outcome of intentional delay in treatment. Gynecologic Oncology, 87 (2), 185-189.

Vliet, V. W., Loon, A. J., Hoor, K. A. \& Boonstra, H. 1998. Cervical carcinoma during pregnancy: Outcome of planned delay in treatment. European Journal of Obstetrics \& Gynecology and Reproductive Biology, 79 (2), 153-157.

\section{MINICURRÍCULO}

JOSÉ RODOLFO TEIXEIRA DA CUNHA graduando em medicina, Universidade Federal do Maranhão, participou dos projetos de extensão intitulados de "Cuidados Paliativos ao Paciente Oncológico Terminal”; "Atendimento Ambulatorial no Serviço de Dor Oncológica no Instituto Maranhense de Oncologia Aldenora Belo em São Luis-MA" e "Neoplasias malignas induzidas pelo tabagismo: cuidar do paciente, não da doença”.

LAILSON OLIVEIRA DE CASTRO graduando em medicina, Universidade Federal do Maranhão, participou dos projetos de extensão intitulados de "Cuidados 
Paliativos ao Paciente Oncológico Terminal”; “Atendimento Ambulatorial no Serviço de Dor Oncológica no Instituto Maranhense de Oncologia Aldenora Belo em São Luis-MA" e "Neoplasias malignas induzidas pelo tabagismo: cuidar do paciente, não da doença”.

MARIA DO DESTERRO SOARES BRANDÃO NASCIMENTO possui graduação em Medicina pela Universidade Federal do Maranhão (1977). Mestrado em Medicina Tropical pela Universidade Federal de Goiás (1986), doutorado em Medicina pela Universidade Federal de São Paulo (1996) e estágio pós-doutoral pelo Programa de Pós-Graduação Stricto Sensu em Oncologia/INCA (2012). Atualmente é professor Titular da Universidade Federal do Maranhão, docente do curso de doutorado da Rede Nordeste de Biotecnologia, coordenadora do Programa de Pósgraduação Saúde do Adulto (PPGSAD) da UFMA e Bolsista de Produtividade em Pesquisa - Modalidade Doutor Sênior / Fundação de Amparo à Pesquisa e ao Desenvolvimento Científico e Tecnológico do Maranhão FAPEMA.

RODRIGO LOPES DA SILVA possui graduação em Medicina pela Universidade Federal do Maranhão (2005). Residência em Cirurgia Oncológica no Hospital Erasto Gaertner - PR (2008-2011). MBA em Gestão de Saúde pela FGV (2016). Mestrado em Saúde do Adulto UFMA (2018). Professor do curso de Medicina do UNICEUMA. Preceptor do internato do curso de Medicina da UEMA. Atualmente trabalha como cirurgião oncológico no CACON Instituto Maranhense de Oncologia Aldenora Belo - IMOAB (MA), onde também é o coordenador do Registro Hospitalar de Câncer (RHC).

ANA BEATRIZ MELO GUIMARÃES graduada em farmácia, pelo Centro universitário UNINTA, pós-graduação em farmácia clínica e prescrição farmacêutica, atualmente residente em Urgência e Emergência na Santa Casa de Misericórdia de Sobral. 